\title{
Kybernetika
}

\section{Michael McAleer}

Stationarity and invertibility of a dynamic correlation matrix

Kybernetika, Vol. 54 (2018), No. 2, 363-374

Persistent URL: http://dml.cz/dmlcz/147200

\section{Terms of use:}

(C) Institute of Information Theory and Automation AS CR, 2018

Institute of Mathematics of the Czech Academy of Sciences provides access to digitized documents strictly for personal use. Each copy of any part of this document must contain these Terms of use.

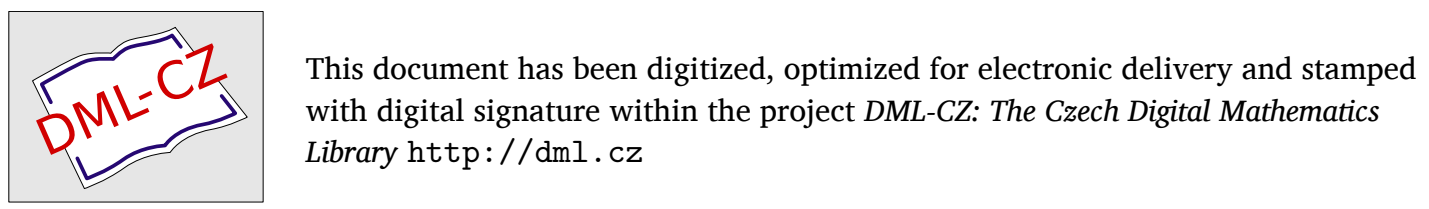




\title{
STATIONARITY AND INVERTIBILITY OF A DYNAMIC CORRELATION MATRIX
}

\author{
Michael McAleer
}

One of the most widely-used multivariate conditional volatility models is the dynamic conditional correlation (or DCC) specification. However, the underlying stochastic process to derive DCC has not yet been established, which has made problematic the derivation of asymptotic properties of the Quasi-Maximum Likelihood Estimators (QMLE). To date, the statistical properties of the QMLE of the DCC parameters have purportedly been derived under highly restrictive and unverifiable regularity conditions. The paper shows that the DCC model can be obtained from a vector random coefficient moving average process, and derives the stationarity and invertibility conditions of the DCC model. The derivation of DCC from a vector random coefficient moving average process raises three important issues, as follows: (i) demonstrates that DCC is, in fact, a dynamic conditional covariance model of the returns shocks rather than a dynamic conditional correlation model; (ii) provides the motivation, which is presently missing, for standardization of the conditional covariance model to obtain the conditional correlation model; and (iii) shows that the appropriate ARCH or GARCH model for DCC is based on the standardized shocks rather than the returns shocks. The derivation of the regularity conditions, especially stationarity and invertibility, may subsequently lead to a solid statistical foundation for the estimates of the DCC parameters. Several new results are also derived for univariate models, including a novel conditional volatility model expressed in terms of standardized shocks rather than returns shocks, as well as the associated stationarity and invertibility conditions.

Keywords: dynamic conditional correlation, dynamic conditional covariance, vector random coefficient moving average, stationarity, invertibility, asymptotic properties

Classification: C22, C52, C58, G32

\section{INTRODUCTION}

Among multivariate conditional volatility models, the dynamic conditional correlation (or DCC) specification of Engle [15] is one of the most widely used in practice. Both multivariate conditional correlations and the associated conditional covariance models are very useful for determining optimal hedging strategies, volatility spillovers and causality in volatility among financial commodities. Checking the underlying stochastic properties, where they might exist, is crucial in examining the internal consistency of

DOI: 10.14736/kyb-2018-2-0363 
the models, as well as in deriving asymptotic properties of the associated parameter estimates, for purposes of sensible empirical analysis.

These theoretical issues are especially important in empirical energy finance, where the relationships among the prices, returns and volatility of fossil fuels, such as oil, coal and gas, and the associated carbon emissions, are crucial for public and private policy making. In this context, Chang and McAleer [6], Chang, McAleer and Tansuchat [7, 8, 9, 10, Chang, McAleer and Zuo [12, and Chang, McAleer and Wang 11 have discussed important practical issues arising in empirical finance, especially as they relate to the pricing, returns and volatility of the primary sources of fossil fuel energy output, the resulting volatility in pricing carbon emissions, and in related stock prices.

In order to calculate optimal hedging strategies (or risk insurance) to mitigate financial risk, the two alternative models that have been used widely for estimating and forecasting multivariate conditional correlations and conditional covariances have been based on: (i) the diagonal and full BEKK models of Baba et al. [3] and Engle and Kroner [16], which have been derived from an $m$-dimensional vector random coefficient autoregressive process (see McAleer et al. 21] and Section 2 below); and (ii) the DCC model, which was presented without an underlying stochastic specification in Engle [15].

The basic DCC modelling approach has been as follows: (i) estimate the univariate conditional variances using the $\operatorname{GARCH}(1,1)$ model of Bollerslev [4, which are based on the returns shocks; and (ii) estimate what is purported to be the conditional correlation matrix of the standardized residuals.

The first step in the modelling approach is arbitrary as the conditional variances could just as easily be based on the standardized residuals themselves, as will be shown in Section 4 below. The second step is fatally flawed as the model can be derived from an appropriate underlying stochastic process as a conditional covariance model rather than as a conditional correlation model. However, as no regularity conditions were presented in the presentation of the DCC model in Engle [15, no statistical properties have yet been derived for the estimated parameters of the model.

A similar comment applies to the varying conditional correlation model of Tse and Tsui [23], where the first stage is based on a standard $\operatorname{GARCH}(1,1)$ model using returns shocks. The second stage is slightly different from the DCC formulation as the conditional correlations are defined appropriately. However, as no regularity conditions are presented, including invertibility, no statistical properties can be derived.

The DCC model has been analyzed critically in a number of papers as its underlying stochastic process has not yet been established, which has made problematic the derivation of the asymptotic properties of the Quasi-Maximum Likelihood Estimators (QMLE). To date, the statistical properties of the QMLE of the DCC parameters have been derived under highly restrictive and unverifiable regularity conditions, which in essence amounts to proof by assumption.

This paper shows that the DCC specification can be obtained from a vector random coefficient moving average process, and derives the sufficient conditions for stationarity and invertibility of the DCC model. The derivation of regularity conditions may subsequently lead to a solid statistical foundation for the estimates of the DCC parameters.

The derivation of DCC from a vector random coefficient moving average process raises three important issues: (i) demonstrates that DCC is, in fact, a dynamic conditional 
covariance model of the returns shocks rather than a dynamic conditional correlation model; (ii) provides the motivation, which is presently missing, for standardization of the conditional covariance model to obtain the conditional correlation model; and (iii) shows that the appropriate ARCH or GARCH model for DCC is based on the standardized shocks rather than the returns shocks.

The remainder of the paper is organized as follows. In Section 2, the standard ARCH model is derived from a random coefficient autoregressive process to provide a background for the remainder of the paper. The multivariate counterpart of $\mathrm{ARCH}$ is derived from a vector random coefficient autoregressive process, which will explain intuitively how the univariate results of Marek [20] on a random coefficient moving average process can be extended to an m-dimensional vector counterpart. In Section 3, the DCC model is presented and discussed. Section 4 presents and discusses a new vector random coefficient moving average process that will be used as an underlying stochastic process in order to derive DCC. Several new results are derived for the associated univariate models, including a novel conditional volatility model expressed in terms of standardized shocks rather than returns shocks, as well as the associated stationarity and invertibility conditions. In Section 5, DCC is demonstrated to be derived from the vector random coefficient moving average process. The conditions for stationarity and invertibility of DCC are derived in Section 6. Some concluding comments are given in Section 7.

\section{RANDOM COEFFICIENT AUTOREGRESSIVE PROCESS}

This section presents the underlying stochastic autoregressive processes for univariate and multivariate GARCH processes, as compared with the multivariate moving average process for the multivariate DCC process in the following section. Consider the following random coefficient autoregressive process of order one:

$$
\varepsilon_{t}=\phi_{t} \varepsilon_{t-1}+\eta_{t}
$$

where

$$
\begin{aligned}
\phi_{t} & \sim i i d(0, \alpha), \\
\eta_{t} & \sim i i d(0, \omega), \text { independent of }\left\{\phi_{t}\right\} .
\end{aligned}
$$

The ARCH(1) model of Engle [14] can be derived as (see Tsay [22]):

$$
h_{t}=E\left(\varepsilon_{t}^{2} \mid I_{t-1}\right)=\omega+\alpha \varepsilon_{t-1}^{2},
$$

where $h_{t}$ is conditional volatility, and $I_{t-1}$ is the information set at time $t$ - 1 . The use of an infinite lag length for the random coefficient autoregressive process in equation (1) leads to the Generalized ARCH (or GARCH) model of Bollerslev [4].

The diagonal version of the BEKK model of Baba et al. 3] and Engle and Kroner [16, though not the Hadamard BEKK and full BEKK models, can be derived from a vector random coefficient autoregressive process (see McAleer et al. [21]). As the statistical properties of vector random coefficient autoregressive processes are well known, the statistical properties of the parameter estimates of the ARCH, GARCH, and diagonal BEKK models are straightforward to establish. 


\section{DCC SPECIFICATION}

This section presents the DCC model, as given in Engle [15, which does not have an underlying stochastic specification that leads to its derivation. Let the conditional mean of financial returns be given as:

$$
y_{t}=E\left(y_{t} \mid I_{t-1}\right)+\varepsilon_{t},
$$

where $y_{t}=\left(y_{1 t}, \ldots, y_{m t}\right)^{\prime}, y_{i t}=\Delta \log P_{i t}$ represents the log-difference in stock prices $\left(P_{i t}\right)$, $i=1, \ldots, m, I_{t-1}$ is the information set at time $t$ - 1 , and $\varepsilon_{t}$ is conditionally heteroskedastic. Without distinguishing between dynamic conditional covariances and dynamic conditional correlations, Engle [15] presented the DCC specification as:

$$
Q_{t}=(1-\alpha-\beta) \bar{Q}+\alpha \eta_{t-1} \eta_{t-1}^{\prime}+\beta Q_{t-1},
$$

where $Q_{t}$ in $(4)$ is purported to be a conditional correlation matrix, without proof, $\bar{Q}$ is assumed to be positive definite with unit elements along the main diagonal, the scalar parameters $\alpha$ and $\beta$ are assumed to be non-negative and satisfy the stability condition, $\alpha+\beta<1$, the standardized shocks, $\eta_{t}=\left(\eta_{1 t}, \ldots, \eta_{m t}\right)^{\prime}$, where $\eta_{i t}=\varepsilon_{i t} / \sqrt{h_{i t}}$, are assumed to be $i i d$, and $D_{t}$ is a diagonal matrix with typical element $\sqrt{h_{i t}}, i=1, \ldots, m$. If $m$ is the number of financial assets, the multivariate definition of the relationship between $\varepsilon_{t}$ and $\eta_{t}$ is $\varepsilon_{t}=D_{t} \eta_{t}$.

Define the conditional covariance matrix of $\varepsilon_{t}$ as $Q_{t}$. As the $m \times 1$ vector, $\eta_{t}$, is assumed to be iid for all $m$ elements, the conditional correlation matrix of $\eta_{t}$ is given by $\Gamma_{t}$. Therefore, the conditional expectation of the covariance matrix of $\varepsilon_{t}$ is defined as:

$$
Q_{t}=D_{t} \Gamma_{t} D_{t}
$$

Equivalently, the conditional correlation matrix, $\Gamma_{t}$, is defined as:

$$
\Gamma_{t}=D_{t}^{-1} Q_{t} D_{t}^{-1} \text {. }
$$

Equation (5) is useful if a model of $\Gamma_{t}$ is available for purposes of estimating $Q_{t}$, whereas equation (6) is useful if a model of $Q_{t}$ is available for purposes of estimating $\Gamma_{t}$. Ling and McAleer [19] and McAleer et al. 21] provide general proofs of the asymptotic properties of univariate and multivariate conditional volatility models based on satisfying the regularity conditions in Jeantheau [18 for consistency, and in Theorem 4.1.3 in Amemiya 2] for asymptotic normality.

In view of equations (5) and (6), as the matrix $Q_{t}$ in equation (4) does not satisfy the definition of a correlation matrix, Engle [15] uses the following standardization for $Q_{t}$ in equation 4 :

$$
R_{t}=\left(\operatorname{diag}\left(Q_{t}\right)\right)^{-1 / 2} Q_{t}\left(\operatorname{diag}\left(Q_{t}\right)\right)^{-1 / 2}
$$

There is no clear explanation given in Engle 15. for the standardization in equation (7) or, more recently, in Aielli [1], especially as equation (7) does not satisfy the definition of a correlation matrix, which is given in equation (6). The standardization in equation (7) might make sense if the matrix $Q_{t}$ in equation (4) were the conditional covariance matrix of $\varepsilon_{t}$ or $\eta_{t}$ though this is not made clear. It is worth noting that the unconditional covariance matrix of $\varepsilon_{t}$ is not analytically tractable. 
Despite the title of the paper, Aielli 1 also does not provide any stationarity conditions for the DCC model, and does not mention invertibility. Indeed, in the literature on DCC, it is not clear whether equation (4) refers to a conditional covariance or a conditional correlation matrix, although the latter is presumed, without proof. Some caveats regarding DCC are given in Caporin and McAleer [5].

\section{VECTOR RANDOM COEFFICIENT MOVING AVERAGE PROCESS}

The random coefficient moving average process will be presented in its original univariate form in section 4.1, as in Marek [20, with an extension to its multivariate counterpart in section 4.2 , in order to derive the corresponding univariate and multivariate conditional volatility models, respectively.

\subsection{Univariate process}

In an interesting and useful paper, Marek [20] proposed a linear moving average model with random coefficients (RCMA), and established the conditions for stationarity and invertibility. In this section, we extend the univariate results of Marek [20] using an mdimensional vector random coefficient moving average process of order $p$, which is used as an underlying stochastic process to derive the DCC model, and prove the stationarity and invertibility conditions. Several new results are also derived for the associated univariate models, including a novel conditional volatility model expressed in terms of standardized shocks rather than returns shocks, as well as the associated stationarity and invertibility conditions.

Consider a univariate random coefficient moving average process given by:

$$
\varepsilon_{t}=\theta_{t} \eta_{t-1}+\eta_{t}
$$

where $\eta_{t} \sim$ iid $(0, \omega)$. The sequence $\left\{\theta_{t}\right\}$ is supposed to be independent of $\eta_{t-1}, \eta_{t}, \eta_{t+1}, \ldots$, which is called the Future Independence Condition, with mean zero and variance $\alpha$. It is also assumed to be measurable with respect to $I_{t}$, where $I_{t}$ is the information set generated by the random variable $\left\{\varepsilon_{t}, \varepsilon_{t-1, \ldots}\right\}$. Furthermore, it is assumed that the process $\left\{\varepsilon_{t}\right\}$ is stationary and invertible, such that $\eta_{t} \in I_{t}$. For further details, see Marek 20.

Without the measurability assumption on $\left\{\theta_{t}\right\}$ it would be difficult to obtain results on the invertibility of the model. However, an important special case of the model arises when $\left\{\theta_{t}\right\}$ is $i i d$, that is, not measurable with respect to $I_{t}$, in which case the conditional and unconditional expectations of $\varepsilon_{t}$ are zero, and the conditional variance of $\varepsilon_{t}$ is given by:

$$
h_{t}=E\left(\varepsilon_{t}^{2} \mid I_{t-1}\right)=\omega+\alpha \eta_{t-1}^{2}
$$

which differs from the $\mathrm{ARCH}(1)$ model in equation (2) in that the returns shock is replaced by the standardized shock. This is a new result in the conditional volatility literature.

As $\eta_{t} \sim$ iid $(0, \omega)$, the unconditional variance of $\varepsilon_{t}$ is given as:

$$
E\left(h_{t}\right)=(1+\alpha) \omega
$$


The use of an infinite lag length for the random coefficient moving average process in equation (8), with appropriate restrictions on $\theta_{t}$, would lead to a generalized ARCH model that differs from the GARCH model of Bollerslev [4] as it would replace the returns shock with a standardized shock.

The univariate ARCH $\sqrt{1}$ model in equation $(9)$ is contained in the family of GARCH models proposed by Hentschel [17, and the augmented GARCH model class of Duan 13 .

It can be shown from the results in Marek [20] that a sufficient condition for stationarity is that the vector sequence $v_{t}=\left(\eta_{t}, \theta_{t} \eta_{t-1}\right)^{\prime}$ is stationary. Moreover, by Lemma 2.1 of Marek 20, a new sufficient condition for invertibility is that:

$$
E\left[\log \left|\theta_{t}\right|\right]<0 \text {. }
$$

The stationarity of $\nu_{t}=\left(\eta_{t}, \theta_{t}, \eta_{t-1}\right)$ and the invertibility condition in equation 10 are new results for the univariate $\mathrm{ARCH}(1)$ model given in equation (9), as well as its direct extension to GARCH models.

\subsection{Multivariate process}

Extending the analysis given above to the multivariate case and to a vector random coefficient moving average (RCMA) model of order $p$, we can derive a special case of $\operatorname{DCC}(p, q)$, namely $\operatorname{DCC}(p, 0)$, as follows:

$$
\varepsilon_{t}=\sum_{j=1}^{p} \theta_{j t} \eta_{t-j}+\eta_{t}
$$

where $\varepsilon_{i}$ and $\eta_{i}$ are both $m \times 1$ vectors and $\theta_{j t}, j=1, \ldots, p$ are random $m \times m$ matrices, independent of $\eta_{t-1}, \eta_{t}, \eta_{t+1}, \ldots$ Under Assumption 1, it is possible to derive the conditional covariance matrix of $\varepsilon_{i}$ in equation (11):

\section{Assumption 1.}

1. $E\left(\eta_{t} \mid I_{t-1}\right)=0, E\left(\eta_{t} \eta_{t}^{\prime} \mid I_{t-1}\right)=\Omega$.

2. The random coefficient matrices $\theta_{j t}$ have the following properties: For all $j=1, \ldots, p$, and $t=1, \ldots, T$, it is assumed that $E\left(\theta_{j t} \mid I_{t-1}\right)=0$ and $E\left(\theta_{j t, k l} \theta_{j t, m n}^{\prime} \mid I_{t-1}\right)=$ $A_{j, k l} A_{j, m n}^{\prime}$ for appropriate matrices $A_{j, k l}$ and $A_{j, m n}$ that form the conditional covariance matrix of $\theta_{j t}$, and $E\left(\theta_{j t, k l} \theta_{i s, m n}^{\prime} \mid I_{t-1}\right)=0, i \neq j$, and/or $s \neq t$.

This is similar to Proposition 1 of McAleer et al. 21] in that the conditional covariance matrix is given by:

$$
H_{t}=E\left(\varepsilon_{t} \varepsilon_{t}^{\prime} \mid I_{t-1}\right)=\Omega+\sum_{j=1}^{p} A_{j} \eta_{t-j} \eta_{t-j}^{\prime} A_{j}^{\prime}
$$

such that:

$$
E\left(\operatorname{vec}\left(H_{t}\right)\right)=\left(I_{m}+\sum_{j=1}^{p} A_{j} \otimes A_{j}\right) \operatorname{vec}(\Omega)
$$


This approach can easily be extended to include autoregressive terms. For example, in a model analogous to $\operatorname{GARCH}(p, q)$, namely:

$$
H_{t}=\Omega+\sum_{i=1}^{p} A_{i} \eta_{t-i} \eta_{t-i}^{\prime} A_{i}^{\prime}+\sum_{j=1}^{q} B_{j} H_{t-j} B_{j}^{\prime},
$$

where the parameter matrices $B_{j}$ are such that the maximum eigenvalue of $\sum_{j=1}^{q} B_{j} \otimes B_{j}$ is smaller than one in modulus, it follows that:

$$
E\left(\operatorname{vec}\left(H_{t}\right)\right)=\left(I_{m}-\sum_{j=1}^{q} B_{j} \otimes B_{j}\right)^{-1}\left(I_{m}+\sum_{j=1}^{p} A_{j} \otimes A_{j}\right) \operatorname{vec}(\Omega)
$$

The derivation given above shows that, as compared with the standard DCC formulation, which is not based on an underlying stochastic process that leads to its derivation, the formulation given above permits straightforward computation of the unconditional variances and covariances via the derived models in equations .

It should also be noted that in Aielli's [1] variation of the standard DCC model, it is possible to calculate the unconditional expectation of the $Q_{t}$ matrix, as in equation (4), but this is not equal to the unconditional covariance matrix of $\varepsilon_{t}$, which is analytically intractable. This is an additional advantage of using the vector random coefficient moving average process given in the above equations, as will be shown explicitly in the following section

\section{DERIVATION OF DCC}

In this section, the DCC model will be derived from a vector random coefficient moving average process as the underlying stochastic process. If $\theta_{j t}$ in equation (11) is given as:

$$
\theta_{j t}=\lambda_{j t} I_{m}, \text { with } \lambda_{j t} \sim \operatorname{iid}\left(0, \alpha_{j}\right)
$$

$j=1, \ldots, p$, where $\lambda_{j t}$ is a scalar random variable, then the conditional covariance matrix can be shown to be:

$$
H_{t}=E\left(\varepsilon_{t} \varepsilon_{t}^{\prime} \mid I_{t-1}\right)=\Omega+\sum_{j=1}^{p} \alpha_{j} \eta_{t-j} \eta_{t-j}^{\prime}
$$

The DCC model in equation (4) is obtained by letting $p \rightarrow \infty$ in equations (11) and (12), setting $\alpha_{j}=\alpha \beta^{j-1}$, and standardizing $H_{t}$ in equation 12 to obtain a conditional correlation matrix. For the case $p=1$ in equation $(12)$, the appropriate univariate conditional volatility model is given in the new model in equation (9), which uses the standardized shocks, rather than standard ARCH in equation (2), which uses the returns shocks.

The derivation of DCC in equation 12 from a vector random coefficient moving average process is important as it: (i) demonstrates that DCC is, in fact, a dynamic conditional covariance model of the returns shocks rather than a dynamic conditional 
correlation model; (ii) provides the motivation, which is presently missing, for standardization of the conditional covariance model to obtain the conditional correlation model; and (iii) shows that the appropriate ARCH and GARCH models for DCC are based on the standardized shocks rather than the returns shocks. Point (iii) provides novel univariate $\mathrm{ARCH}$ and GARCH models.

It is worth noting that the derivation of the DCC model using the underlying vector random coefficient moving average process is not argued to be unique as the latter has not been shown to be a necessary condition. However, to date there has been no derivation of the DCC model from an underlying stochastic process that leads to its derivation.

\section{DERIVATION OF STATIONARITY AND INVERTIBILITY OF DCC}

The formulation of DCC given in the previous section is more natural than the standard treatment as it can be derived from an underlying stochastic process which leads to its derivation, and can be also analyzed in terms of mathematical and statistical properties, such as stationarity, invertibility, and existence of moments.

This section derives the stationarity and invertibility conditions for the DCC model in Theorem 1, based on Assumption 2:

\section{Assumption 2.}

$$
E\left[\log \left\|\Theta_{t-k}\right\|\right]<\log \sqrt{p m}
$$

where $\left\|\Theta_{t}\right\|$ is the Frobenius norm, and $\Theta_{t}$ is given by:

$$
\Theta_{t}=\left(\begin{array}{cccc}
-\theta_{1 t} & -\theta_{2 t} & \ldots & -\theta_{p t} \\
1 & 0 & \ldots & 0 \\
. & . & . & . \\
0 & \ldots & 1 & 0
\end{array}\right)
$$

Theorem 1. A sufficient condition for stationarity is that the vector sequence:

$$
v_{t}=\left(\eta_{t}, \theta_{1 t} \eta_{t-1}, \ldots, \theta_{p t} \eta_{t-p}\right)^{\prime}
$$

is stationary. Furthermore, under Assumption 2, the vector random coefficient moving average process, $\varepsilon_{t}$, is invertible.

Proof. The proof of stationarity is similar to the sufficient condition for stationarity of the univariate random coefficient moving average process, namely that the vector sequence $v_{t}=\left(\eta_{t}, \theta_{t} \eta_{t-1}\right)^{\prime}$ is stationary. For invertibility, note that:

$$
\eta_{t}=\varepsilon_{t}-\sum_{j=1}^{p} \theta_{j t} \eta_{t-j}
$$

which can be written as:

$$
\tilde{\eta}_{t}=\Theta_{t} \tilde{\eta}_{t-1}+\tilde{\varepsilon}_{t}
$$


where $\tilde{\eta}_{t}=\left(\eta_{t}, \eta_{t-1}, \ldots, \eta_{t-p+1}\right)^{\prime}$ and $\tilde{\varepsilon}_{t}=\left(\varepsilon_{t}, \varepsilon_{t-1}, \ldots, \varepsilon_{t-p+1}\right)^{\prime}$.

Hence,

$$
\tilde{\eta}_{t}=\sum_{j=0}^{n-1}\left(\prod_{k=1}^{j} \Theta_{t-k+1}\right) \tilde{\varepsilon}_{t-j}+\left(\prod_{k=0}^{n-1} \Theta_{t-k}\right) \tilde{\eta}_{t-n} .
$$

Now let:

$$
\tilde{\eta}_{t}^{(n)}=\sum_{j=0}^{n}\left(\prod_{k=1}^{j} \Theta_{t-k+1}\right) \tilde{\varepsilon}_{t-j}
$$

Consider

$$
\begin{gathered}
\frac{1}{n} \log \frac{1}{\sqrt{p m}}\left\|\tilde{\eta}_{t}-\tilde{\eta}_{t}^{n}\right\|=\frac{1}{n} \log \frac{1}{\sqrt{p m}}\left\|\left(\prod_{k=1}^{n-1} \Theta_{t-k}\right) \tilde{\eta}_{t-n}\right\| \\
\leq \frac{1}{n} \log \frac{1}{\sqrt{p m}}\left\|\prod_{k=1}^{n-1} \Theta_{t-k}\right\|+\frac{1}{n} \log \frac{1}{\sqrt{p m}}\left\|\tilde{\eta}_{t-n}\right\| \\
\leq \frac{1}{n} \sum_{k=1}^{n} \log \frac{1}{\sqrt{p m}}\left\|\Theta_{t-k}\right\|+\frac{1}{n} \log \frac{1}{\sqrt{p m}}\left\|\tilde{\eta}_{t-n}\right\| \\
\underset{a . s .}{\longrightarrow} \log \frac{1}{\sqrt{p m}}\left\|\Theta_{t-k}\right\|<0
\end{gathered}
$$

as $E \log \left\|\Theta_{t-k}\right\|<\sqrt{p m}$, by assumption. This implies that $\eta_{t}-\eta_{t}^{n} \underset{\text { a.s. }}{\longrightarrow} 0$ and, hence, $\eta_{t}$ is asymptotically measurable with respect to $\left\{\varepsilon_{t-1}, \varepsilon_{t-2}, \ldots\right\}$, and $\varepsilon_{t}$ is invertible.

The derivation of the sufficient conditions for stationarity and invertibility of the DCC model in Theorem 1 makes it more viable and understandable in practice, and contributes toward a statistical analysis of the model for practical purposes, as discussed in Section 1.

Note that a sufficient condition for equation 13 is that:

$$
\sum_{j=1}^{p} E\left\|\theta_{j t}\right\|^{2}<m
$$


as

$$
\begin{aligned}
& E \log \frac{1}{\sqrt{p m}}\left\|\Theta_{t-k}\right\| \leq \log E \frac{1}{\sqrt{p m}}\left\|\Theta_{t-k}\right\| \\
= & \log E \frac{1}{\sqrt{p m}} \sqrt{\sum_{j=1}^{p}\left\|\theta_{j t}\right\|^{2}+(p-1) m} \\
= & \log E \sqrt{\frac{1}{\sqrt{p m}} \sum_{j=1}^{p}\left\|\theta_{j t}\right\|^{2}+(p-1) / p} \\
\leq & \log \sqrt{\frac{1}{\sqrt{p m}} \sum_{j=1}^{p} E\left\|\theta_{j t}\right\|^{2}+(p-1) / p} \\
< & 0 .
\end{aligned}
$$

The condition given in equation (14) may be easier to check in practice than the condition given in equation (13). The simplicity and convenience of equation (13) may be important for the practical implementation of the DCC model.

For the special case $\theta_{j t}=\lambda_{j t} I_{m}$, with $\lambda_{j t} \sim i i d\left(0, \alpha_{j}\right), j=1, \ldots, p$, discussed in Section 5 above, the condition in equation (14) simplifies to the well-known condition on the long-run persistence to returns shocks, namely:

$$
\sum_{j=1}^{p} E \lambda_{j t}^{2}=\sum_{j=1}^{p} \alpha_{j}<1 .
$$

\section{CONCLUSION}

The paper was concerned with one of the most widely-used multivariate conditional volatility models, namely the dynamic conditional correlation (or DCC) specification. As the underlying stochastic process to derive the DCC model has not yet been established, this has made problematic the derivation of the asymptotic properties of the Quasi-Maximum Likelihood Estimators (QMLE). To date, the statistical properties of the QMLE of the DCC parameters have been derived under highly restrictive and unverifiable regularity conditions.

The paper showed that the DCC specification could be obtained from a vector random coefficient moving average process, and derived the sufficient stationarity and invertibility conditions of the DCC model. The derivation of the regularity conditions may eventually lead to a solid foundation for the statistical analysis of the QMLE estimates of the DCC parameters.

The derivation of DCC demonstrated that DCC is, in fact, a dynamic conditional covariance model of the standardized shocks rather than a dynamic conditional correlation model based on returns shocks, as presumed in Engle [15]. Moreover, the derivation of the DCC model provided the motivation, which is presently missing, for standardizing the conditional covariance model to obtain the conditional correlation model. The standardization of the estimated DCC models in practice does not satisfy the definition of a 
correlation matrix, which has always been problematic in interpreting the DCC model (see, for example, Caporin and McAleer [5]).

The derivation of the DCC model also showed that the appropriate ARCH and GARCH models underlying the DCC model are based on the standardized shocks rather than the returns shocks. Several new results were also derived for univariate models, including a novel conditional volatility model that was derived from an underlying univariate random coefficient moving average process.

\section{ACKNOWLEDGEMENT}

The author is most grateful to Gian Piero Aielli, Massimiliano Caporin, Chialin Chang, Guillaume Gaetan Martinet, Yuk Tse, the Editor and two referees of Kybernetika, and especially Christian Hafner, for very helpful comments and suggestions. For financial support, the author wishes to acknowledge the Australian Research Council, Ministry of Science and Technology (MOST), Taiwan.

(Received April 9, 2017)

\section{REFERENCES}

[1] G. P. Aielli: Dynamic conditional correlations: On properties and estimation. J. Business Econom. Statist. 31 (2013), 282-299. DOI:10.1080/07350015.2013.771027

[2] T. Amemiya: Advanced Econometrics. Harvard University Press, Cambridge 1985.

[3] Y. Baba, R. F. Engle, D. Kraft, and K. F. Kroner: Multivariate simultaneous generalized ARCH. Unpublished manuscript, Department of Economics, University of California, San Diego 1985, (the published version is given in Engle and Kroner [16]).

[4] T. Bollerslev: Generalised autoregressive conditional heteroscedasticity. J. Econometr. 31 (1986), 307-327. DOI:10.1016/0304-4076(86)90063-1

[5] M. Caporin and M. McAleer: Ten things you should know about the dynamic conditional correlation representation. Econometrics 1 (2013), 1, 115-126. DOI:10.3390/econometrics1010115

[6] C.-L. Chang and M. McAleer: A simple test for causality in volatility. Econometrics 5 (2017), 1, 5 pp. DOI:10.3390/econometrics5010015

[7] C.-L. Chang, M. McAleer, and R. Tansuchat: Modelling conditional correlations for risk diversification in crude oil markets. J. Energy Markets $2(2009 / 10), 4,1-23$. DOI:10.2139/ssrn.1401331

[8] C.-L. Chang, M. McAleer, and R. Tansuchat: Analyzing and forecasting volatility spillovers, asymmetries and hedging in major oil markets. Energy Economics 32 (2010), 1445-1455. DOI:10.1016/j.eneco.2010.04.014

[9] C.-L. Chang, M. McAleer, and R. Tansuchat: Crude oil hedging strategies using dynamic multivariate GARCH. Energy Economics 33 (2011), 5, 912-923. DOI:10.1016/j.eneco.2011.01.009

[10] C.-L. Chang, M. McAleer, and R. Tansuchat: Conditional correlations and volatility spillovers between crude oil and stock index returns. North Amer. J. Econom. Finance 25 (2013), 116-138. DOI:10.1016/j.najef.2012.06.002 
[11] C.-L. Chang, M. McAleer, and Y.-A. Wang: Modelling volatility spillovers for bioethanol, sugarcane and corn spot and futures prices. Renewable Sustainable Energy Rev. 81 (2018), 1, 1002-1018. DOI:10.1016/j.rser.2017.07.024

[12] C.-L. Chang, M. McAleer, and G. D. Zuo: Volatility spillovers and causality of carbon emissions, oil and coal spot and futures for the EU and USA. Sustainability 9 (2017), 10, p. 1789, 1-22. DOI:10.3390/su9101789

[13] J.-C. Duan: Augmented $\operatorname{GARCH}(p, q)$ process and its diffusion limit. J. Econometrics 79 (1997), 97-127. DOI:10.1016/s0304-4076(97)00009-2

[14] R. F. Engle: Autoregressive conditional heteroscedasticity with estimates of the variance of United Kingdom inflation. Econometrica 50 (1982), 987-1007. DOI:10.2307/1912773

[15] R. F. Engle: Dynamic conditional correlation: A simple class of multivariate generalized autoregressive conditional hereoskedasticity models. J. Business Econom. Statist. 20 (2002), 339-350.

[16] R. F. Engle and K. F. Kroner: Multivariate simultaneous generalized ARCH. Econometr. Theory 11 (1995), 122-150. DOI:10.1017/s0266466600009063

[17] L. Hentschel: All in the family: Nesting symmetric and asymmetric GARCH models. J. Financial Economics 39 (1995), 71-104. DOI:10.1016/0304-405x(94)00821-h

[18] T. Jeantheau: Strong consistency of estimators for multivariate ARCH models. Econometr. Theory 14 (1998), 70-86. DOI:10.1017/s0266466698141038

[19] S. Ling and M. McAleer: Asymptotic theory for a vector ARMA-GARCH model. Econometr. Theory 19 (2003), 280-310. DOI:10.1017/S0266466603192092

[20] T. Marek: On invertibility of a random coefficient moving average model. Kybernetika 41 (2005), 01, 743-756.

[21] M. McAleer, F. Chan, S. Hoti, and O. Lieberman: Generalized autoregressive conditional correlation. Econometr. Theory 24 (2008), 6, 1554-1583. DOI:10.1017/s0266466608080614

[22] R. S. Tsay: Conditional heteroscedastic time series models. J. Amer. Statist. Assoc. 82 (1987), 590-604. DOI:10.2307/2289470

[23] Y.K. Tse and A.K. C. Tsui: A multivariate GARCH model with time-varying correlations. J. Business Econom. Statist. 20 (2002), 351-362. DOI:10.1198/073500102288618496

Michael McAleer, Department of Finance, Asia University, Taiwan, and Discipline of Business Analytics, University of Sydney Business School, Australia, and Econometric Institute, Erasmus School of Economics, Erasmus University Rotterdam, The Netherlands, and Department of Economic Analysis and ICAE, Complutense University of Madrid, Spain, and Institute of Advanced Sciences, Yokohama National University, Japan.

e-mail: michael.mcaleer@gmail.com 